\title{
Uncorrectable Dimensions in Congenital Canal Atresia Making Us to Revise Operative Candidacy and Patient Selection
}

\section{Wael A Alzamil*}

Otorhinolaryngology, Hearing and Speech Institute, Cairo, Egypt

*Corresponding Author: Wael A Alzamil, Otorhinolaryngology, Hearing and Speech

Institute, Cairo, Egypt.
Received: July 10, 2021

Published: July 22, 2021

(C) All rights are reserved by Wael A Alzamil.

\section{Abstract}

Background: Congenital aural atresia is common birth defects and is often associated with auricular and middle ear deformities. The external auditory canal anomaly varies from slightly narrow to complete atresia. Congenital external auditory canal stenosis is defined by Jahrsdoerfer., et al. as an EAC with a diameter of less than $4 \mathrm{~mm}$ [1]. The surgical management for canal atresia and stenosis is aimed to improve hearing and establish an appropriate auditory canal status for possible application of hearing aids. In clinical practice, we found that patients with canal stenosis and partial atresia have a better postoperative hearing improvement and fewer postoperative complications than those with complete canal atresia [2]. By analyzing the temporal CT images and intraoperative findings in patients with canal atresia and comparing those with images and intraoperative findings from normal canals, we further understand external and middle ear development to improve surgical candidacy selection and avoid unnecessary complications [3]. In addition to the status of the ossicular chain, the degree of development and pneumatization of the tympanic cavity and mastoid, and the course of the facial nerve, the most important information taken from CT and intraoperative findings are the dimensions of the proposed future canal. There are main three dimensions which are the lateral and medial canal diameters and the depth of the canal. Each diameter has two main diagonals, the antero posterior dimension and the supero inferior dimension. This study will focus on the dimensions of the created canal or cavity and whether it is correctable or uncorrectable.

Aim of the Study: To match the C.T. scan and intraoperative findings with the surgical outcome for patients with congenital canal atresia and illustrate the operated uncorrectable dimensions with unfavorable outcome for later proper patient selection and surgical candidacy considerations.

Patients and Methods: A prospective, study conducted from September 2017 to December 2020 on 50 patients suffering congenital canal atresia. Patients have been subjected to preoperative audiological assessment and high resolution C.T. scanning. All patients addressing the study have been scored according to the Jahrsdoerfer Grading Scale selecting patients with scores above 6 (50 patients). All patients have undergone surgical meatocanaloplasty via anterior approach or posterior approach according to the C.T. findings. Intraoperative data collection was done to be matched with preoperative C.T. data and correlated later on with the final outcome. Postoperative regular follow up visits were done obligatory every two to three weeks for at least six months and for regular packing. Results: Data collection was done involving intraoperative anatomical findings, difficulties or complications, immediate and late postoperative results including the final outcome. Intraoperative measurements have showed marked reduction of the depth of the cavity or the created canal ranging from 1 to $1.5 \mathrm{~cm}$ in 47 operated ears having complete atresia and more favorable depth ranging from 1.75 to $2.25 \mathrm{~cm}$ in the last three patients with C.T. evidence of partial atresia and less affected tympanic ring. Regarding the antero-posterior distance of the cavity, it was doubled and tripled after radical mastoidectomy in the 47 patients of complete atresia 
whoever in the three partial atresia patients who have operated via the anterior approach the dimension was equal to the normal auditory canal $(0.7-1 \mathrm{~cm})$. The supero-inferior dimension was markedly less even after Atticotomy in the 47 patients undergoing radical mastoidectomy with a range of $1-1.5 \mathrm{~cm}$. It measured $0.7-1 \mathrm{~cm}$ in the three patients of partial atresia. No major intraoperative complications were recorded except one 4 years old female who suffered ipsilateral immediate postoperative complete facial paralysis who recovered completely after 4 months. Follow up of all patients recorded progressive circumferential narrowing of their canals and progressive reduction their depth. The final outcome showed shallow canals in 48 patients of depth not more than $1.2 \mathrm{~cm}$ and less shallow canals in two patients with their depth $2 \mathrm{~cm}$.

Conclusion: Strict analysis of the C.T. data and intraoperative findings in patients of congenital canal atresia proves their inoperability due to the uncorrectable dimensions even for those patients with the highest Jahrsdoerfer score due to lack of full dimension consideration.

Keywords: Uncorrectable Canal Dimensions; Congenital Canal Atresia

\section{Introduction}

By the end of the $4^{\text {th }}$ week of development, the auricle develops from 6 mesenchymal proliferations/swellings known as hillocks derived from the first and second pharyngeal arches that surround the first pharyngeal cleft. There are six auricular hillocks on each side of the external meatus that eventually fuse to form the auricle. The external ears begin their embryological development in the lower neck region and gradually ascend posterolaterally to the level of the eyes as the mandible develops [4].

By the thirteenth week, a disk-like plug comes into contact with the primordial malleus medially, contributing to the future formation of the tympanic membrane. By the fifteenth week, the disk-like plug splits, leaving behind a thin ectodermal cell layer of the immature tympanic membrane. A continuation of the thin skin of the pinna lines the entire external auditory canal and the outer surface of the tympanic membrane. The external auditory canal is completely patent and expands to its complete form by the eighteenth week [5].

The temporal bone consists of four parts, the squamous, mastoid, petrous and tympanic parts. The squamous part is the largest and most superiorly positioned relative to the rest of the bone. The zygomatic process is a long, arched process projecting from the lower region of the squamous part and it articulates with the zygomatic bone. Poster inferior to the squamous is the mastoid part. Fused with the squamous and mastoid parts and between the sphenoid and occipital bones lies the petrous part, which is shaped like a pyramid [6]. The tympanic part is relatively small and lies inferior to the squamous part, anterior to the mastoid part, and superior to the styloid process. The styloid, from the Greek stylos, is a phallic shaped pillar directed inferiorly and anteromedially between the parotid gland and internal jugular vein [7].

The temporal bone is ossified from eight centers, exclusive of those for the internal ear and the tympanic ossicles: one for the squama including the zygomatic process, one for the tympanic part, four for the petrous and mastoid parts, and two for the styloid process. Just before the end of prenatal development [8]. The temporal bone consists of three principal parts.

The squama is ossified in membrane from a single nucleus, which appears near the root of the zygomatic process about the second month.

The petromastoid part is developed from four centers, which make their appearance in the cartilaginous ear capsule about the fifth or sixth month. One (proötic) appears in the neighborhood of the eminentia arcuata, spreads in front and above the internal auditory meatus and extends to the apex of the bone; it forms part of the cochlea, vestibule, superior semicircular canal, and medial wall of the tympanic cavity. A second (opisthotic) appears at the promontory on the medial wall of the tympanic cavity and surrounds the fenestra cochleæ; it forms the floor of the tympanic cavity and vestibule, surrounds the carotid canal, invests the lateral and lower part of the cochlea, and spreads medially below the internal auditory meatus. A third (pterotic) roofs in the tympanic cavity and 
antrum; while the fourth (epiotic) appears near the posterior semicircular canal and extends to form the mastoid process (Vrolik) [9].

The tympanic ring is an incomplete circle, in the concavity of which is a groove, the tympanic sulcus, for the attachment of the circumference of the eardrum (tympanic membrane). This ring expands to form the tympanic part, and is ossified in membrane from a single center which appears about the third month. The styloid process is developed from the proximal part of the cartilage of the second branchial or hyoid arch by two centers: one for the proximal part, the tympanohyal, appears before birth; the other, comprising the rest of the process, is named the stylohyal, and does not appear until after birth. The tympanic ring unites with the squama shortly before birth; the petromastoid part and squama join during the first year, and the tympanohyal portion of the styloid process about the same time. The stylohyal does not unite with the rest of the bone until after puberty, and in some skulls never at all [10].

Regarding postnatal development, apart from size increase, the chief changes from birth through puberty in the temporal bone are as follows.

The tympanic ring extends outward and backward to form the tympanic part. This extension does not, however, take place at an equal rate all around the circumference of the ring, but occurs more at its anterior and posterior portions. As these outgrowths meet, they create a foramen in the floor of the canal, the foramen of Huschke. This foramen is usually closed about the fifth year, but may persist throughout life [11].

The mandibular fossa is at first extremely shallow and looks lateral and inferior; it deepens and directs more inferiorly over time. The part of the squama which forms the fossa lies at first below the level of the zygomatic process. As, the base of the skull thickens, this part of the squama is directed horizontal and inwards to contribute to the middle cranial fossa, and its surfaces look upward and downward; the attached portion of the zygomatic process everts and projects like a shelf at a right angle to the squama [12].

The mastoid portion is at first flat, with the stylomastoid foramen and rudimentary styloid immediately behind the tympanic ring. With air cell development, the outer part of the mastoid component grows anteroinferiorly to form the mastoid process, with the styloid and stylomastoid foramen now on the under surface.
The descent of the foramen is accompanied by a requisite lengthening of the facial canal [13].

The downward and forward growth of the mastoid process also pushes forward the tympanic part; as a result, its portion that formed the original floor of the canal, and contained the foramen of Huschke, rotates to become the anterior wall. The fossa subarcuata is nearly effaced [14].

The external auditory canal is derived from the first branchial cleft after resorption of the ectodermal thickening leaving a thin layer of ectoderm (skin or stratified squamous epithelium) lining the canal and tympanic membrane. The cartilaginous and bony portions of the canal are derived from the mesoderm of the first and second branchial arches. The outer cartilaginous one third measures about 8 - $12 \mathrm{mms}$ and is continuous with the pinna cartilages. The inner bony two thirds measure about $16-20 \mathrm{mms}$ and is composed of the tympanic ring, squamous portion and mastoid portion superiorly and posteriorly respectively [15].

Surgery for congenital aural atresia usually has unfavorable prognosis. So authors have developed a grading schemes based on the preoperative temporal bone CT scan and the appearance of the external ear. Jahrsdoerfer scoring system was the most popular one in which patients are graded on a possible best score of 10 [16]. The stapes is assigned the highest rating ( 2 points), while all other entrees on the scale are 1 point table 1 . The grade assigned preoperatively has been supposed to correlate well with the patient's chance of success regarding postoperative speech reception threshold of 15 to $25 \mathrm{~dB}$. Patients with a preoperative grade of $8 / 10$ would, therefore, have a 80 percent chance of achieving this threshold. Patients with scores of $5 / 10$, or less, are not considered surgical candidates, as the risk of the operation would outweigh the possible benefits. The grading system should avoid non indicated surgeries allowing proper prediction of the hearing outcome. Whoever in our practice different scenarios exists? Prediction of the outcome was not always possible by this grading system. This may be due to other factors or parameters which are not involved in the grading system or may be as a result of uncorrectable factors which means inoperability [17].

So proper understanding of the embryology and the development of the external auditory canal should alter the concept of meatal atresia from just failure of canalization to agenesis of the 
canal. The external auditory canal is not a negative structure, whoever it can be considered an organ with its own constituents and dimensions. So, in those patients with canal atresia the bony and cartilaginous walls are partially or completely absent. Their developmental absence will raise the idea of creating the canal rather than reconstructing it. Creation of the auditory canal means that we should imitate its normal dimensions including its depth and its diameter. Regarding the normal auditory canal length or depth it measures about 2.5 centimeters ( 1 inch) in length (from the tragus to the tympanic membrane) and 0.7 centimeters ( 0.3 inch) in diameter and is curved in an $\mathrm{S}$ shape direction. The mandibular condyle sits anterior to the bony portion of the external acoustic canal and the mastoid air cells sit behind the bony portion of the canal [18]. The parotid salivary gland lies anterior and inferior to the cartilaginous portion of the canal. Again, in patients with canal atresia we find the distance between the tragus and the outer wall of the middle ear to be shorter than normal $(2.5 \mathrm{cmms})$ due to underdevelopment of bony and cartilaginous constituents (Figure 1). Beside this shallow distance the depth of the surrounding tissues is reduced as a result of the hypoplasia affecting the first and second branchial arches derivatives such as the temporomandibular joint, temporalis muscle and the parotid region. All these facts regarding morbid anatomy will definitely limit any surgeon to correct the depth or the length of the canal because the middle ear is near to the surface [19].

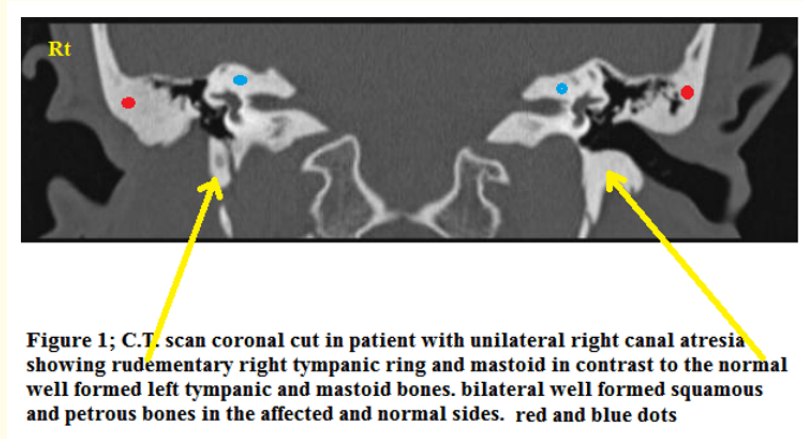

The normal external canal has nearly a cylindrical skeleton or framework formed of bone and cartilage. For proper correction of canal atresia we should imitate this cylindrical skeleton to keep the canal patent which never to happen. During surgery for canal atresia either with the anterior approach or with the posterior ap- proach (radical mastoidectomy) we cannot obtain a cylindrical or hollow solid canal [20]. Only we get a shallow superior and posterior bony boundaries due to hypoplastic mastoid and squamous bones and very short anterior and inferior bony boundaries due to rudimentary tympanic ring and temporomandibular joint. In addition, there is absent cartilaginous portion of the canal due to rudimentary cartilaginous vestiges which cannot form a solid cylinder. So, beside the shallow cavity or proposed canal there is defects in the solid boundaries of the canal to be replaced by the surrounding soft tissues (Figure 2). These defects will definitely lead to later collapse and contraction of the created cavity or canal beside the gradual process of fibrosis and healing by secondary intention leading to more narrow and shallow cavity [21].

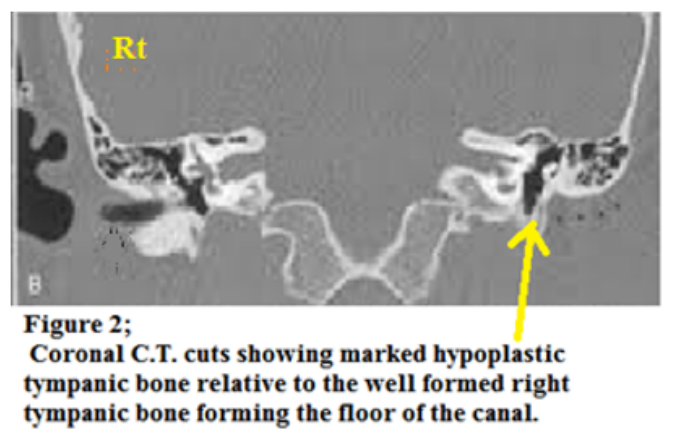

As regards the caliber of the canal, it has a diameter range from 0.7 to $1 \mathrm{~cm}$ in its antero-posterior and supero-inferior dimensions. This diameter is nearly equal along the canal from lateral to medial except the isthmus narrowing at the junction of the cartilaginous and bony portions of the canal. Before atresia surgery we usually look for the thickness of the atretic plate and the distance between the sigmoid sinus and the temporomandibular joint to measure the antero-posterior dimension [22]. Sometimes we cannot differentiate between the atretic plate and the mastoid due to partially cellular plate. In rare mild cases with incomplete agenesis the atretic plate has a fair antero-posterior dimension (not less than $1 \mathrm{~cm}$ ) which enable us to perform the anterior approach by removing the atretic plate. Whoever, in the majority of patients there is narrow plate forcing us to perform the posterior approach in which we do radical mastoidectomy to maximize the antero-posterior distance between the sigmoid sinus and the temporomandibular joint [23]. 
Regarding the other diagonal which is the supero-inferior dimension, it is usually overlooked or omitted during the preoperative evaluation. This dimension is very important and critical. The superior bony canal wall in the inner two thirds is formed by squamous portion of the temporal bone and its zygomatic process forming what is called the outer attic mass or the lateral boundary of the attic region. The inferior wall is formed of the floor of the tympanic ring. In cases of complete canal atresia, the superior wall is less affected whoever the inferior wall is short in its width and acquires a higher level due to underdeveloped tympanic ring leading to marked decrease in the vertical or supero-inferior dimension. Intraoperative drilling of the outer attic mass removing it (Atticotomy) may increase this dimension but to a limit which may be insufficient (Figure 3 and 4). So drilling is limited superiorly by the dura and inferiorly by the soft tissue, parotid or anomalous facial nerve course with the rudimentary tympanic ring. As regards the cartilaginous outer one third, in cases of complete atresia with anotia or grade III microtia there will be rudimentary malformed cartilage vestiges which cannot be utilized to reconstruct the canal. In my study, I have revised in details the C.T. data and comparing it with the intraoperative findings and the postoperative coarse regarding the healing process and hearing improvement.

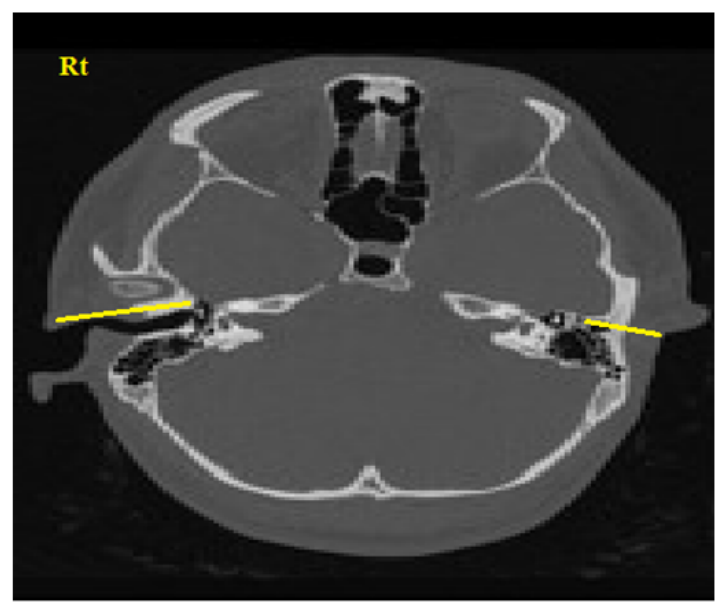

Figure 3: Axial C.T. cut showing the marked differrence in depth between right normal side and the left atretic side marked by the yellow lines. this reflects the shallow left side as a whole leading to uncorrectable depth.

\section{Patients and Methods}

A prospective, study conducted from September 2017 to December 2020 on 50 patients suffering congenital canal atresia. 36

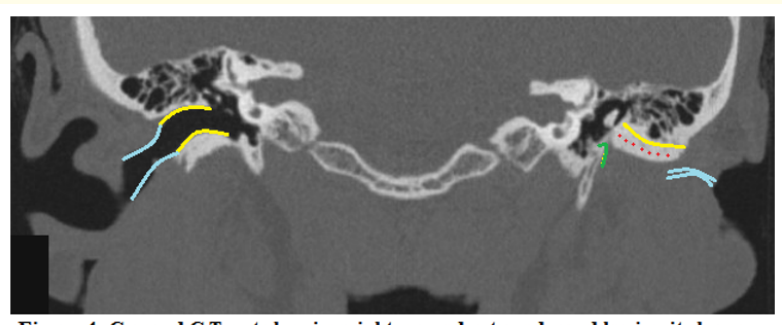

Figure 4: Coronal C.T. cut showing right normal external canal having its bony part marked by the yellow lines and cartilagenous part marked by the blue lines. the left atretic ear showes only the superior bony wall marked by the yellow line with the atretic plate under it marked by red dots and rudimentary inferior bony wall due to hypoplastic tympanic ring marked by the green line. Also in the left side we notice the aproximate site of the malformed cartilage marked by the blue line.

patients have bilateral meatal atresia and 14 patients with unilateral atresia. Age distribution has a range of $4-18.5$ years old with a main of 8 years. Sex distribution showed slight male predominance by 28 male patients $56 \%$ versus 22 female patients $44 \%$. Patients have been subjected to detailed history taking and thorough general and local examination to identify possible perinatal risk factors or relevant family history and to exclude other congenital anomalies which may interfere with surgery. All patients in the study were subjected to preoperative audiological assessment and high resolution C.T. scanning. Regarding the audiological assessment was done by pure tone audiometry (PTA) for patients with age equal and more than 7 years. PTA showed conductive hearing loss in the affected ears with a gap of $40-55 \mathrm{db}$ with a main of $48 \mathrm{db}$. For patients less than 7 years old, free field audiometry (FFA) and auditory brain stem response (ABR) were the assessment tool. FFA and $\mathrm{ABR}$ were done additionally to three patients with age more than 7 due to unreliable PTA and low IQ. All patients in the study have a Jahrsdoerfer scores above 6. All patients have undergone surgical meatocanaloplasty via anterior approach or posterior approach according to the C.T. findings. Intraoperative data collection was done to be matched with preoperative C.T. data and correlated later on with the final outcome. Postoperative regular follow up visits were done regularly every two to three weeks for at least six months and for regular packing.

\section{Ethics}

The ethics committee of our institution approved this work according to the Declaration of Helsinki. A detailed informed consent was obtained from each patient. 


\section{Operative procedure}

Under general endotracheal anesthesia with patient supine, head elevated and tilted to the opposite side. Draping and sterilization was done then we perform surface markings to identify the temporomandibular joint, zygomatic process and the mastoid tip. Local infiltration anesthesia using saline adrenaline in a concentration of $1 / 200000$ to be injected in the post auricular sulcus and over the temporal region. According to the development and location of the auricle we plan the skin incision which was in the majority of patients in the post auricular sulcus. After periosteal elevation we start to localize certain anatomical landmarks to adjust our drilling in the proper location. The most important and nearly constant landmarks are the temporomandibular joint (the anterior limit) and the zygomatic root which marks the dural level (the superior limit). According to the preoperative C.T. scan findings we select the surgical approach. In 47 patients $94 \%$ we found narrow atretic plate with marked underdevelopment of the tympanic ring and mastoid bone and so we proceeded through the posterior approach by doing modified radical mastoidectomy to maximize the antero-posterior dimension. We started at the anterosuperior angle between the line of the temporomandibular joint and the line of dural plate which is marked by the zygomatic process of the squamous bone and the zygomatic bone. Drilling in the atretic plate to reach dural plate superiorly and the TMJ anteriorly by thinning the bone or egg shelling it to be marked by slight bluish color. Drilling at this angle will remove the outer attic mass by doing Atticotomy to get access to the epitympanum to find the incudomalleal complex or the fused head of malleus and mal directed bulky incus. Then we continue drilling posteriorly to exenterate mastoid air cells to reach the sigmoid sinus and sinodural angle. We lower the facial ridge taking care of possible anomalous facial nerve course. After that we continue drilling inferiorly in the rudimentary tympanic ring. We found that inferior drilling is markedly limited by several factors. The first factor was the markedly hypoplastic tympanic ring in its width and height and the small sized mesotympanum and hypotympanum. The second factor is the anomalous facial nerve which may acquire more anterior position. In three patients $6 \%$ who have a C.T. evidence of wide adequate atretic plate and less hypoplastic tympanic ring and mastoid process, we performed the anterior approach by removing the atretic plate only without mastoidectomy. Also with this approach we start drilling at the anterosuperior angle as the safest point by performing Atticotomy removing the outer attic mass then we proceed posteriorly and inferiorly to reach a suitable canal diameter usually $2 \mathrm{~cm}$ in the vertical dimension and $1.5 \mathrm{~cm}$ in its horizontal dimension. After obtaining the bony canal or cavity in both approaches we drill any fine bone prominence near by the ossicular chain to ensure its free mobility and avoid fixation by new bone formation. Then we apply a temporalis fascial graft over the ossicular chain which is usually hypermobile and splinting the graft by Gelfoam in the middle ear cavity and over the graft. The next step was meatoplasty. To obtain a wide meatus we excise soft tissue and cartilaginous remnants opposite the created canal to reach double or triple its normal diameter $(1.5-2.5 \mathrm{~cm})$ and to compensate the suspected postoperative stenosis. We try to centralize the created meatus opposite the created canal by anchoring sutures to the periosteum in three different directions and antibiotic soaked gauze packing. Partial thickness Thiersch skin grafting was done in some patients to cover the bare bone of the canal.

Postoperative management was in the form of parenteral broad spectrum antibiotics for five days followed by oral antibiotics for five days. Postoperative analgesia for one week. The antibiotic soaked gauze pack was removed after two weeks to replaced by a new pack. Regular pack changing every two to three weeks will take place for a period of four to six months. The first three or four packs in the follow up period was antibiotic soaked gauze pack then we start to use dry merocel packing and wet it by antibiotic drops to swell and double its size. All patients were advised to use antibiotic drops twice daily on the merocel pack. Merocel packing was an essential and pivotal step due to its stored static power of expansion which lead to gradual dilatation and canal widening. Merocel packing was avoided only intraoperatively and early postoperatively to prevent marked reaction and granulations.

\section{Results}

Data collection was done involving intraoperative anatomical findings, difficulties or complications, immediate and late postoperative results including the final outcome. Intraoperative measurements have showed marked reduction of the depth of the cavity or the created canal ranging from 1 to $1.5 \mathrm{~cm}$ in 47 operated ears with complete atresia and more favorable depth ranging from 1.75 to $2.25 \mathrm{~cm}$ in the last three patients with C.T. evidence of partial atresia and less affected tympanic ring. Regarding the anteroposterior distance of the cavity, it was doubled and tripled after radical mastoidectomy in the 47 patients of complete atresia who- 
ever in the three partial atresia patients who have operated via the anterior approach the dimension was equal to the normal auditory canal $(0.7-1 \mathrm{~cm})$. The supero-inferior dimension was markedly less even after Atticotomy in the 47 patients undergoing radical mastoidectomy with a range of $1-1.5 \mathrm{~cm}$. It measured $0.7-1 \mathrm{~cm}$ in the three patients of partial atresia. No major intraoperative complications were recorded except one 4 years old female who suffered ipsilateral immediate postoperative complete facial paralysis which regained its complete recovery after 4 months.

Follow up of all patients recorded progressive circumferential narrowing of their canals and progressive reduction their depth. The final outcome showed shallow canals in 48 patients of depth not more than $1.2 \mathrm{~cm}$ and less shallow canals in two patients with their depth $2 \mathrm{~cm}$.

In my study I have noticed gradual decrease in hearing level postoperatively to be stable after 4-6 months. The postoperative gap has a range of $15-28 \mathrm{db}$ with a mean of $20 \mathrm{db}$.

\section{Discussion}

Surgical repair for congenital aural atresia remains one of the most challenging tasks in our practice. Atresia surgery has multiple risks and unpredictable postoperative scenarios regarding hearing and healing results. The risks of atresia repair include injury to the facial nerve, sensorineural hearing loss, failure to close the air-bone gap, and meatal or canal stenosis [24]. The goals of atresia repair are to obtain the best possible hearing outcome and to construct a clean, wide, well epithelialized external ear canal which may adapt a hearing aid mold and also to obtain a tympanic membrane [25].

Surgical candidacy and patient selection depends on several factors and parameters which have been formulated by many authors to construct scoring systems. Jahrsdoerfer scoring system was considered the most popular and useful among others. Based on the evaluation of certain anatomical structures on high-resolution computed tomography of the temporal bone, the Jahrsdoerfer grading system was developed to determine surgical candidacy and to predict which patients would have more favorable hearing outcomes. Previous studies demonstrated that patients scoring 6 or lower have a worse postoperative hearing outcome than patients scoring 7 or higher [26].

In a study, lack of middle ear aeration was the only variable evaluated by the Jahrsdoerfer grading scale that was significantly more correlated with a poorer hearing outcome. This study found no statistically significant difference was noted in the presence or absence of the other 8 anatomical structures evaluated using the Jahrsdoerfer scale between groups, although the facial nerve position has a significance when surgery was aborted in some patients. Lack of aeration may result in difficulty identifying a middle space and ossicular chain during drilling, refixation of the ossicular chain postoperatively, and postoperative stenosis with a constricted middle ear space and smaller tympanic membrane. So, simplification of the Jahrsdoerfer grading scale, or a modified Jahrsdoerfer grading scale was done to determine a patient's candidacy for surgery using computed tomography to assess 4 primary structures that would immediately preclude surgery. These structures are the position of the tegmen, the location and position of the facial nerve, the presence of middle ear aeration, and the appearance of the stapes bone or oval window [27].

In contrast, other studies have attempted to correlate the surgical anatomy and audiometric outcome with other preoperative factors such as age, severity of microtia, and revision vs. primary surgery. They found that the surgical outcome does not correlate with the middle ear aeration only or other middle ear structures which were mentioned in Jahrsdoerfer or modified Jahrsdoerfer grading scale A. Similarly, in my study I have noticed that the outcome could not be predicted precisely by the Jahrsdoerfer scoring system in which there was recurrences of stenosis and hearing loss with the highest scores and well developed middle ear and ossicles. I have analyzed the preoperative C.T. scan data and matching it with the intraoperative findings [28]. Then I have followed those patients closely through their postoperative months to pick up the gradual and progressive changes. For us to construct the atretic canal properly we have to get a scoring scale for the canal itself, not the middle ear only. Other previous scales have focused on the middle ear space and its contents and the shape or the development of the auricle bypassing the canal development and its dimensions [29]. Reconstruction of the canal is not just burrowing through the obstructing tissues but it should be in the form of building the canal to mimic its components as possible. The external canal is a hollow patent structure with bony and cartilaginous solid walls (Figure 7). It has a length and a caliber, so if we are able to mimic these dimensions and construct a conduit from the exterior to the middle ear, the outcome may be favorable. Unfortunately, to the moment some dimensions and components of the canal may be uncorrectable. The superior and posterior bony walls are the only ones which we 
may obtain; the superior bony wall is composed of the squamous portion of the temporal bone which can be widened in the superior direction by drilling out the outer attic mass to perform Atticotomy up to the level of middle fossa dura. The posterior bony wall is formed of the underdeveloped mastoid portion of the temporal bone. It can be widened posteriorly by performing mastoidectomy to the sinus plate especially in patients with narrow atretic plate [30]. The anterior and inferior bony walls are nearly absent due to the rudimentary tympanic ring and temporomandibular joint. The outer cartilaginous third of the canal is absent or replaced by a deformed or stenosed cartilaginous remnants. So, finally the canal will be short or shallow down to its half and the existing canal has only superior and posterior bony walls with opposing inferior and anterior soft tissue walls leading to ultimate collapse and stenosis (Figure 5 and 6). The length or depth of the canal is uncorrectable dimension due to the fact of underdevelopment or no development of this side of the face including the external auditory canal, temporomandibular joint and surrounding soft tissue. So the depth is reduced all over the side of affection whether it is bilateral or unilateral and the middle ear is near to the surface than normal. And that is why we should be careful regarding the facial nerve which will be more superfacial in the underdeveloped mastoid. Also the anterior and inferior bony walls are uncorrectable due to their marked underdevelopment and small size relative to the superior and posterior walls. Additionally, we have another uncorrectable dimension which is the vertical or supero-inferior dimension between the tegmen and the tympanic floor. This dimension is limited superiorly by the dura and inferiorly by the small sized thin tympanic ring which should not be drilled completely to obtain inferior bony wall rather than a soft one and to avoid trauma to the anomalous facial nerve. Dimension consideration is of paramount importance either in patients with complete atresia or in patients with partial atresia or stenosis. These uncorrectable dimensions are nearly constant in all cases even those with the highest Jahrsdoerfer scores. If we reconstruct the anterior and inferior wall by a costal cartilage (for example) to oppose the same height of superior and posterior walls we may get a patent solid canal even may be short or shallow but we did partial correction. Otherwise, I recommend to avoid atresia surgery in the presence of uncorrectable C.T. dimensions. So the scope of patient selection and surgical candidacy will be so narrowed and limited to any patient with suitable dimensions (if present). Repair of unilateral aural atresia was a controversial issue for several decades and may be limited for patients who have reliable preoperative indicators or scores and was usually postponed to early adulthood and to get the patient's own decision being a relative rather than absolute indication. Through my study, I don't recommend repair of unilateral atresia even for adult patients with optimal Jahrsdoerfer scores unless they have near normal C.T. dimensions of the external canal.
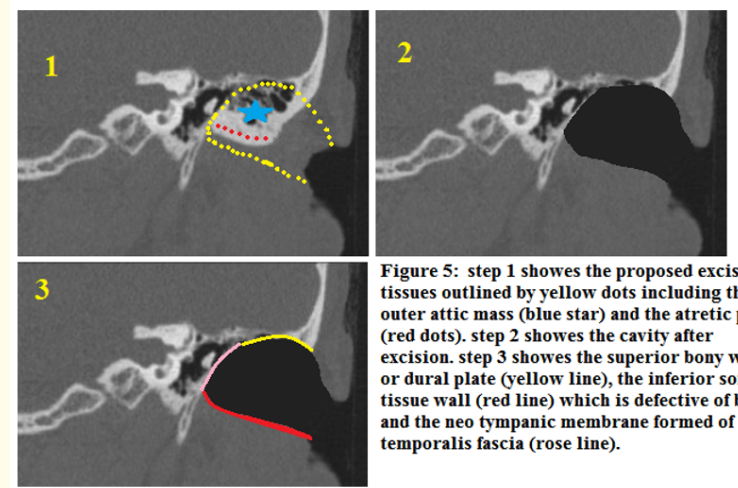

Figure 5: step 1 showes the proposed excised tissues outlined by yellow dots including the outer attic mass (blue star) and the atretic plate (red dots). step 2 showes the cavity after excision. step 3 showes the superior bony wall or dural plate (yellow line), the inferior soft tissue wall (red line) which is defective of bone and the neo tympanic membrane formed of temporalis fascia (rose line).

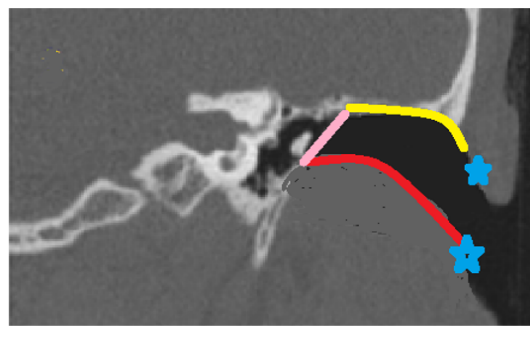

Figure 6 : the cavity in figure 5 was showen here inprogressive narrowing by
the inferior soft tissue wall (red line) and progressive (red line) and progressive narrowing of the meatus
because it is devoid of solid secause it is devoid of solid
skeleton (bone or cartilage) Blue stars
Figure 7

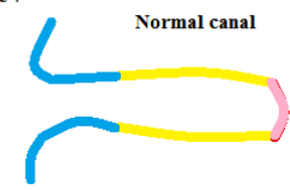

Normal canal depth Inner bony two thirds Outer carilagenous one third continous with the auricular cartilage Normal caliber of the canal

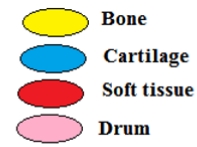


The postoperative audiometry was obtained one 1 month postoperatively and repeated every three months. De la Cruz and Teufer t reported relatively stable hearing with no significant change in the air-bone gap from short- to long-term (6 months) follow-up [31]. A study by Lambert demonstrated some degradation in hearing after 1 year postoperatively [32]. Similarly, in my study I have noticed gradual decrease in hearing level postoperatively to be stable after 4 - 6 months. The degree of hearing loss was not parallel to the degree of recurrent stenosis even with recurrent complete atresia the hearing was fair with an air bone gap less than the preoperative one. This may be due to replacement of the bony atresia by soft tissues.

Conventional hearing aids can be utilized for patients with unsatisfactory postoperative hearing provided there is sufficient depth for the mold. Otherwise bone-oscillating or bone-anchored hearing device are the solution [33].

\section{Conclusion}

Proper analysis of the C.T. data and matching it with the intraoperative findings in patients with congenital canal atresia has proven their inoperability due to the uncorrectable dimensions even for those patients with the highest Jahrsdoerfer score due to lack of full dimension consideration. So I recommend to slow down and not to rush for atresia surgery before we revise thoroughly all dimensions and its feasibility for correction through the high resolution C.T. scan.

\section{Financial Support and Sponsorship}

Nil.

\section{Conflicts of Interest}

There are no conflicts of interest.

\section{Bibliography}

1. Curtin HD. "Congenital malformations of the ear". Otolaryngologic Clinics of North America 21.2 (1988): 317-336.

2. Zhang TY., et al. "Management of congeni $\neg$ tal meatal stenosis and atresia: impact factors on long-term results and surgical strategy". Chinese Journal of Otolaryngology 10.1 (2012): 15-18.

3. Yeakley JW and Jahrsdoerfer RA. "CT evaluation of congenital aural atresia: what the radiologist and surgeon need to know".
Journal of Computer Assisted Tomography 20.5 (1996): 724731.

4. Fuchs JC and Tucker AS. "Development and Integration of the Ear". Current Topics in Developmental Biology 115 (2015): 213232.

5. Anthwal N and Thompson H. "The development of the mammalian outer and middle ear". Journal of Anatomy 228.2 (2016): 217-232.

6. Hall JW. "Development of the ear and hearing". Journal of Perinatology 20.8-2 (2000): S12-20.

7. Giraldez F and Fritzsch B. "The molecular biology of ear development - "Twenty years are nothing". The International Journal of Developmental Biology 51.6-7 (2007): 429-438.

8. Solomon KS., et al. "Genetic interactions underlying otic placode induction and formation". Developmental Dynamics 230.3 (2004): 419-433.

9. Chang W., et al. "The development of semicircular canals in the inner ear: role of FGFs in sensory cristae". Development 131.17 (2004): 4201-4211.

10. Barrionuevo F., et al. "Sox9 is required for invagination of the otic placode in mice". Developmental Biology 317.1 (2008): 213-224.

11. Urness LD., et al. "FGF signaling regulates otic placode induction and refinement by controlling both ectodermal target genes and hindbrain Wnt8a". Developmental Biology 340.2 (2010): 595-604.

12. Hans S., et al. "Fgf-dependent otic induction requires competence provided by Foxi1 and Dlx3b". BMC Developmental Biology 7 (2007): 5.

13. Ohyama T., et al. "Wnt signals mediate a fate decision between otic placode and epidermis". Development 133.5 (2006): 865875.

14. Klockars T and Rautio J. "Embryology and epidemiology of microtia”. Facial Plastic Surgery 25.3 (2009): 145-148.

15. Ikui A., et al. "Postnatal development of the tympanic cavity: a computer-aided reconstruction and measure $\neg$ ment study". Acta Oto-Laryngologica 120.3 (2000): 375-379. 
16. Jahrsdoerfer RA., et al. "Gradᄀing system for the selection of patients with congenital aural atresia". American Journal of Otolaryngology 13.1 (1992): 6-12.

17. Shonka DC Jr., et al. "The Jahrsdoerfer grad ing scale in surgery to repair congenital aural atresia". Archives of Otorhinolaryngology-Head and Neck Surgery 134.8 (2008): 873-877.

18. Tos M. "Anatomy and histology of the middle ear". Clinical Reviews in Allergy and Immunology 2.4 (1984): 267-284.

19. Takegoshi H and Kaga K. "Difference in facial canal anatomy in terms of severity of microtia and deformity of middle ear in patients with microtia”. Laryngoscope 113.4 (2003): 635-639.

20. Dedhia K., et al. "Anatomic variants on computed tomography in congenital aural atresia". Otolaryngology-Head and Neck Surgery 147.2 (2012): 323-328.

21. Osborn AJ., et al. "Middle ear volume as an adjunct measure in congenital aural atresia". International Journal of Pediatric Otorhinolaryngology 75.7 (2011): 910-914.

22. Kelley PE and Scholes MA. "Microtia and congenital aural atresia". Otolaryngologic Clinics of North America 40.1 (2007): 6180.

23. Schuknecht HF. “Congenital aural atresia”. Laryngoscope 99.9 (1989): 908-917.

24. Oliver ER., et al. "Middle ear dimensions in congenital aural atresia and hearing outcomes after atresiaplasty". Otology and Neurotology 31.6 (2010): 946-953.

25. Kountakis SE., et al. "Microtia grade as an indicator of middle ear development in aural atresia". Archives of Otorhinolaryngology-Head and Neck Surgery 121.8 (1995): 885-886.

26. Mukherji SK., et al. "Imag ᄀing of congenital anomalies of the branchial apparatus". Neuroimaging Clinics of North America 10.1 (2000): 75-93.

27. Benton $C$ and Bellet PS. "Imaging of congenital anomalies of the tempo $\neg$ ral bone”. Neuroimaging Clinics of North America 10.1 (2000): 35-53.
28. Fu Y and Zhang T. "Facial nerve lying lateral to ossicles in one case of congenital aural atresia". International Journal of Pediatric Otorhinolaryngology 75.4 (2011): 597-599.

29. Jahrsdoerfer RA., et al. "Surface con 7 tour three-dimensional imaging in congenital aural atresia". Archives of Otorhinolaryngology-Head and Neck Surgery 119.1 (1993): 95-99.

30. Swartz JD and Faerber EN. "Congenital malformations of the external and middle ear: high-resolution CT findings of surgical import". American Journal of Roentgenology 144.3 (1985): 501-506.

31. Yellon RF and Branstetter BF. "Prospective blinded study of comput $\urcorner$ ed tomography in congenital aural atresia". International Journal of Pediatric Otorhinolaryngology 74.11 (2010): 1286-1291.

32. Hill CA and Richtsmeier JT. "A quantitative method for the evaluation of three-dimensional structure of temporal bone pneumatization". Journal of Human Evolution 55.4 (2008): 682-690.

33. Vrabec JT., et al. "3D CT imaging method for measuring temporal bone aeration”. Acta Oto-Laryngologica 122.8 (2002): 831835.

\section{Volume 3 Issue 9 September 2021} (C) All rights are reserved by Wael A Alzamil. 